\title{
On Norm of Elementary Operator: An Application of Stampfli's Maximal Numerical Range
}

\author{
Denis Njue Kingangi \\ Department of Mathematics \& Computer Science, University of Eldoret, Eldoret, Kenya
}

\section{Email address:}

dankingangi2003@yahoo.com

\section{To cite this article:}

Denis Njue Kingangi. On Norm of Elementary Operator: An Application of Stampfli's Maximal Numerical Range. Pure and Applied Mathematics Journal. Vol. 7, No. 1, 2018, pp. 6-10. doi: 10.11648/j.pamj.20180701.12

Received: February 4, 2018; Accepted: March 8, 2018; Published: March 27, 2018

\begin{abstract}
Many researchers in operator theory have attempted to determine the relationship between the norm of an elementary operator of finite length and the norms of its coefficient operators. Various results have been obtained using varied approaches. In this paper, we attempt this problem by the use of the Stampfli's maximal numerical range.
\end{abstract}

Keywords: Elementary Operator, Maximal Numerical Range, Rank-One Operator

\section{Introduction}

Properties of elementary operators have been investigated in the resent past under varied aspects. Their norms have been a subject of interest for research in operator theory. Deriving a formula to express the norm of an arbitrary elementary operator in terms of its coefficient operators remains a topic of research in operator theory. In the current paper, the concept of the maximal numerical range is applied in determining the lower bound of the norm of an elementary operator consisting of two terms, and also to determine the conditions under which the norm of this operator is expressible in terms of its coefficient operators in $B(H)$. Specifically, the Stampfli's maximal numerical range is employed in arriving at our results.

Let $H$ be a complex Hilbert space and $B(H)$ be the set of bounded linear operators on $H$. We define an elementary operator,

$$
E_{n}: B(H) \rightarrow B(H), W \mapsto \sum_{i=1}^{n} T_{i} W S_{i},
$$

for all $W \in B(H)$ where $T_{i}, S_{i}$ are fixed elements of $B(H)$. When $n=1$, then we obtain a basic elementary operator,

$$
E_{n}: B(H) \rightarrow B(H), W \rightarrow T W S,
$$

for all $W \in B(H)$ and $T, S$ fixed in $B(H)$. We denote the basic elementary operator by $M_{T, S}$. When $n=2$, we obtain the elementary operator of length two, whereby,

$$
E_{2}(W)=T_{1} W S_{1}+T_{2} W S_{2},
$$

for all $W \in B(H)$ and $T_{i}, S_{i}$ fixed in $B(H)$ for $i=1,2$.

The Jordan elementary operator, $U_{T, S}$, is defined as;

$$
U_{T, S}: B(H) \rightarrow B(H), W \rightarrow T W S+S W T,
$$

for all $W \in B(H)$ and $T, S$ fixed in $B(H)$, is an example of elementary operators.

Let $\mathcal{A}$ be an algebra. A derivation is a function $\Delta: \mathcal{A} \rightarrow$ $\mathcal{A}$ for which $\Delta(x, y)=x(\Delta y)+(\Delta \mathrm{x}) \mathrm{y}$ for all $x, y \in \mathcal{A}$. If there is an $a \in \mathcal{A}$ such that $\Delta x=x a-a x$ for all $x \in \mathcal{A}$, then $a$ ? ? is called an inner derivation. A derivation is another example of elementary operators.

The Stampfli's maximal numerical range of $T \in B(H)$ is the set,

$W_{0}(T)=\left\{\lambda \in \mathbb{C}:\left\langle T x_{n}, x_{n}\right\rangle \rightarrow \lambda,\left\|x_{n}\right\|=1,\left\|T x_{n}\right\| \rightarrow\|T\|\right\}$,

while the maximal numerical range $W_{S}\left(T^{*} S\right)$ of $T^{*} S$ relative to $S$ is defined as,

$$
\begin{gathered}
W_{S}\left(T^{*} S\right)=\left\{\lambda \in \mathbb{C}: \exists\left\{x_{n}\right\} \subseteq H,\left\|x_{n}\right\|=1,\right. \\
\left.\lim _{n \rightarrow \infty}\left\langle T^{*} S x_{n}, x_{n}\right\rangle=\lambda, \lim _{n \rightarrow \infty}\left\|S x_{n}\right\|=\|S\|\right\},
\end{gathered}
$$
where $T^{*}$ is the Hilbert adjoint of $T$.

For any $x, y \in H$, the rank one operator, $x \otimes y \in B(H)$, is defined by $(x \otimes y)(z)=\langle z, y\rangle x$, for all $z \in H$.

This paper has determined the norm of the elementary operator of length two.

Given and elementary operator $E_{2}$ on $B(H)$ with fixed operators $T_{i}, S_{i}$ on $B(H)$ for $i=1,2$, does the relationship 
$\left\|E_{2}\right\|=\sum_{i=1}^{2}\left\|T_{i}\right\|\left\|S_{i}\right\|$ hold? King'ang'i [1] attempted this problem using the maximal numerical range of $T^{*} S$ relative to $S$. The current paper employs the concept of the Stampfli's maximal numerical range to determine the lower bound of the norm of elementary operator $E_{2}$, and also to determine the conditions under which the norm of this operator is expressible in terms of the norms of its coefficient operators in $B(H)$. The approach used by Barraa and Boumazguor [2], and also by King'ang'i [1] is employed in obtaining our results.

\section{The Norm of the Jordan Elementary Operator}

Let $H$ be a complex Hilbert space, $B(H)$ be the algebra of bounded linear operators on $H$, and $T, S \in B(H)$ be fixed. For a Jordan elementary operator $U_{T, S}$, Mathieu [5], in 1990 proved that in the case of prime $\mathrm{C}^{*}$-algebras, the lower bound of the norm of $U_{T, S}$ can be estimated by

$$
\left\|U_{T, S}\right\| \geq \frac{2}{3}\|T\|\|S\|
$$

In 1994, Cabrera and Rodriguez [3], proved that

$$
\left\|U_{T, S}\right\| \geq \frac{1}{20412}\|T\|\|S\|,
$$

for prime $\mathrm{JB}^{*}$-algebras.

On their part, Stacho and Zalar [6], in 1996, worked on the standard operator algebra (which is a sub-algebra of $B(H)$ that contains all finite rank operators). They first showed that the operator $U_{T, S}$ actually represents a Jordan triple structure of a $C^{*}$-algebra. They also showed that if $A$ is a standard operator algebra acting on a Hilbert space $H$, and $T, S \in A$, then

$$
\left\|U_{T, S}\right\| \geq 2(\sqrt{2}-1)\|T\|\|S\| .
$$

They later (1998), proved that

$$
\left\|U_{T, S}\right\| \geq\|T\|\|S\|
$$

for the algebra of symmetric operators acting on a Hilbert space. They attached a family of Hilbert spaces to standard operator algebra and used the inner products in them to obtain their results.

Barraa and Boumazguor [2], in the year 2001 used the concept of the numerical range of $T$ relative to $S$, denoted by $W_{S}\left(T^{*} S\right)$, to obtain their results. They employed the idea of finite rank operators to prove the following theorem;

Theorem 2.1 Let $H$ be a complex Hilbert space and $B(H)$ be the algebra of all bounded linear operators on $H$. If $T, S \in B(H)$ with $S \neq 0$, then;

$$
\left\|U_{T, S}\right\| \geq \sup _{\lambda \in W_{S}\left(T^{*} S\right)}\left\{\|\| S\left\|T+\frac{\bar{\lambda}}{\|S\|} S\right\|\right\} .
$$

As a consequent of this, they proved the following corollary;

Corollary 2.2 Let $H$ be a complex Hilbert space and T, $S$ be bounded linear operators on $H$. If $0 \in W_{S}\left(T^{*} S\right) \cup W_{T}\left(S^{*} T\right)$, then;

$$
\left\|U_{T, S}\right\| \geq\|T\|\|S\| .
$$

They also proved the following proposition;

Proposition 2.3 Let $H$ be a complex Hilbert space and $T, S$ be bounded linear operators on $H$. If $\|T\|\|S\| \in$ $W_{T}\left(S^{*} T\right) \cap \in W_{T^{*}}\left(S T^{*}\right)$, then;

$$
\left\|U_{T, S}\right\|=2\|T\|\|S\| .
$$

Proves to theorems 2.1, 2.2 and 2.3, can be obtained from [2].

\section{Norm of Elementary Operator of Length Two}

Kingangi et al [4] in 2014 used finite rank operators to determine the norm of the elementary operator $E_{2}$. Below is the theorem they proved (see theorem 2.5):

Theorem 3.1 Let $H$ be a complex Hilbert space and $B(H)$ be the algebra of all bounded linear operators on $H$. Let $E_{2}$ be the elementary operator on $B(H)$ defined above. If for an operator $W \in B(H)$ with $\|W\|=1$ we have $W(x)=x$ for all unit vectors $x \in H$, then;

$$
\left\|E_{2}\right\|=\sum_{i=1}^{2}\left\|T_{i}\right\|\left\|S_{i}\right\|,
$$

for $i=1,2$.

For the proof of theorem 3.1, see King' ang'i [4], theorem 2.5 .

Odero et al [7], determined the norm of tensor product elementary operator. They showed that for an inner derivation $\Delta_{T}$, we have $\left\|\Delta_{T}\right\|=2\|T\|$ if and only if $0 \in$ $W(T)$. They also proved that if $S, T \in B(H)$, then we have;

$$
\left\|E_{2}\right\|=\sup \{\|T X-X S\|: X \in B(H),\|X\|=1\} .
$$

Jocic et al [8], proved that if $\left\{T_{\alpha}\right\}_{\alpha \in \Lambda}$ and $\left\{S_{\alpha}\right\}_{\alpha \in \Lambda}$ are weakly*-measurable families of bounded Hilbert space operators such that transfers $X \mapsto \int_{\Lambda} T_{\alpha}^{*} X T_{\alpha} d \mu(\alpha)$ and $X \mapsto \int_{\Lambda} S_{\alpha}^{*} X S_{\alpha} d \mu(\alpha)$ on $B(H)$ have their spectra contained in the unit disc, then for all bounded operators $X$, we have;

$$
\left\|\Delta_{T} X \Delta_{S}\right\| \leq\left\|X-\int_{\Lambda} T_{\alpha}^{*} X S_{\alpha} d \mu(\alpha)\right\|,
$$

where

$$
\Delta_{T}=S-\lim _{r>1}\left(I+\sum_{n=1}^{\infty} r^{2 n} \int_{\Lambda} \ldots \int_{\Lambda}\left|T_{\alpha_{1}} \ldots T_{\alpha_{n}}\right|^{2} d \mu^{n}\left(\alpha_{1}, \ldots, \alpha_{n}\right)\right)^{-\frac{1}{2}}
$$


and $\Delta_{S}$ by analogy.

Wafula, et al [9], considered normally represented elementary operators. They proved that the norm of an elementary operator is equal to the largest singular value of the operator itself. They also proved that, if the Jordan elementary operator $U_{T, S}$, we have;

$$
\left\|U_{T, S}\right\|_{i n j} \geq 2 \sqrt{2-l}\|T\|\|S\|
$$

where $S, T \in B(H)$.

In 2017, King'ang'i [1] employed the concept of the maximal numerical range of $T^{*} S$ relative to $S$ to determine the lower norm of an elementary operator of length two. He proved the following theorem (see theorem 3.1):

Theorem 3.2. Let $E_{2}$ be an elementary operator of length two on $B(H)$. Then,

$$
\left\|E_{2}\right\| \geq \sup _{\lambda \in W_{S_{1}}\left(S_{2}{ }^{*} S_{1}\right)}\left\{\|\| S_{1}\left\|T_{1}+\frac{\bar{\lambda}}{\left\|S_{1}\right\|} T_{2}\right\|\right\},
$$

where $S_{i}, T_{i}$ are fixed elements of $B(H)$ for $i=1,2$.

He also determined the conditions on which the norm of $E_{2}$ is expressible in terms of the norms of its coefficient operators by proving the following theorems (see corollary 3.2 and theorem 3.3):

Corollary 3.3 Let $H$ be a complex Hilbert space and $T_{i}, S_{i}$ be bounded linear operators on $H$ for $i=1,2$. Let $0 \in$ $W_{S_{1}}\left(S_{2}^{*} S_{1}\right) \cup W_{S_{2}}\left(S_{1}^{*} S_{2}\right)$. Then, $\left\|E_{2}\right\| \geq\left\|T_{1}\right\|\left\|S_{1}\right\|$, where $E_{2}$ is as defined earlier.

Theorem 3.4 Let $H$ be a complex Hilbert space and $T_{i}, S_{i}$ be bounded linear operators on $H$ for $i=1,2$. Let $E_{2}$ be an elementary operator of length two. If $\left\|T_{1}\right\|\left\|T_{2}\right\| \in W_{T_{1}^{*}}\left(T_{2} T_{1}^{*}\right)$ and $\left\|S_{1}\right\|\left\|S_{2}\right\| \in W_{S_{2}}\left(S_{1}^{*} S_{2}\right)$, then, $\left\|E_{2}\right\|=\sum_{i=1}^{2}\left\|T_{i}\right\|\left\|S_{i}\right\|$.

Below, we present more results on the norm of this operator by employing the concept of theStampfli's maximal numerical range. In theorem 3.5, we determine the lower bound of the norm of the operator $E_{2}$ while in theorem 3.6 we determine the conditions necessary to express the norm of $E_{2}$ in the form $\left\|E_{2}\right\|=\sum_{i=1}^{2}\left\|T_{i}\right\|\left\|S_{i}\right\|$.

Theorem 3.5 Let $E_{2}$ be an elementary operator on $B(H)$ and $S_{1}, S_{2} \in B(H)$. If $\lambda_{i} \in W_{0}\left(S_{i}\right)$ for each $\lambda_{i} \in \mathbb{C}, i=1,2$, then we have $\left\|E_{2}\right\| \geq \sup _{\lambda_{i} \in W_{0}\left(S_{i}\right)}\left\{\left\|\sum_{i=1}^{2} \lambda_{i} T_{i}\right\|: T_{i} \in\right.$ $B(H), i=1,2\}$.

Proof. Let $\left\{x_{n}\right\}_{n \geq 1}$ be a sequence of unit vectors in a Hilbert space $H$ and $y \otimes x_{n} \in B(H)$ be a rank-one operator on $H$ for a unit vector in $y \in H$, defined by $\left(y \otimes x_{n}\right)(x)=$ $\left\langle x, x_{n}\right\rangle y$ for all $x \in H$. Recall the Stampfli' smaximal numerical range of $T \in B(H)$ is the set,

$W_{0}(T)=\left\{\lambda \in \mathbb{C}:\left\langle T x_{n}, x_{n}\right\rangle \rightarrow \lambda,\left\|x_{n}\right\|=1,\left\|T x_{n}\right\| \rightarrow\|T\|\right\}$,

If $\lambda_{1} \in W_{0}\left(S_{1}\right)$ and $\lambda_{2} \in W_{0}\left(S_{2}\right)$, then there are sequences $\left\{x_{n}\right\}_{n \geq 1}$ and $\left\{y_{n}\right\}_{n \geq 1}$ of unit vectors in $H$ such that $\lim _{n \rightarrow \infty}\left\langle S_{1} x_{n}, x_{n}\right\rangle=\lambda_{1} \quad, \quad \lim _{n \rightarrow \infty}\left\|S_{1} x_{n}\right\|=\left\|S_{1}\right\| \quad$ and $\lim _{n \rightarrow \infty}\left\langle S_{2} x_{n}, x_{n}\right\rangle=\lambda_{2}, \lim _{n \rightarrow \infty}\left\|S_{2} x_{n}\right\|=\left\|S_{2}\right\|$.

Now, we have;

$$
\begin{aligned}
& \left\|\left(E_{2}\left(y \otimes x_{n}\right)\right) x_{n}\right\|=\left\|\left(\sum_{i=1}^{2} M_{T_{i}, S_{i}}\left(y \otimes x_{n}\right)\right) x_{n}\right\| \\
& \leq\left\|\sum_{i=1}^{2} M_{T_{i}, S_{i}}\left(y \otimes x_{n}\right)\right\|\left\|x_{n}\right\| \\
& \leq\left\|\sum_{i=1}^{2} M_{T_{i}, s_{i}}\right\|\left\|y \otimes x_{n}\right\| \\
& \leq\left\|\sum_{i=1}^{2} M_{T_{i}, S_{i}}\right\|\|y\|\left\|x_{n}\right\| \\
& =\left\|\sum_{i=1}^{2} M_{T_{i}, s_{i}}\right\|
\end{aligned}
$$

Therefore,

$$
\begin{aligned}
& \left\|\sum_{i=1}^{2} M_{T_{i}, S_{i}}\right\| \geq\left\|\left(\sum_{i=1}^{2} M_{T_{i}, S_{i}}\left(y \otimes x_{n}\right)\right) x_{n}\right\| \\
& =\left\|\left(T_{1}\left(y \otimes x_{n}\right) S_{1}+T_{2}\left(y \otimes x_{n}\right) S_{2}\right) x_{n}\right\| \\
& =\left\|T_{1}\left(y \otimes x_{n}\right) S_{1} x_{n}+T_{2}\left(y \otimes x_{n}\right) S_{2} x_{n}\right\| \\
& =\left\|\left\langle S_{1} x_{n}, x_{n}\right\rangle T_{1} y+\left\langle S_{2} x_{n}, x_{n}\right\rangle T_{2} y\right\|
\end{aligned}
$$

Taking limits as $n \rightarrow \infty$, we obtain;

$$
\left\|\sum_{i=1}^{2} M_{T_{i}, S_{i}}\right\| \geq\left\|\lambda_{1} T_{1} y+\lambda_{2} T_{2} y\right\|=\left\|\sum_{i=1}^{2} \lambda_{i} T_{i} y\right\|,
$$

and this is true for any $\lambda_{1} \in W_{0}\left(S_{1}\right)$ and $\lambda_{2} \in W_{0}\left(S_{2}\right)$, and for any unit vector $y \in H$.

Since $\lambda_{i} \in W_{0}\left(S_{i}\right)$ for $i=1,2$, and $y \in H$ are arbitrarily chosen, then we obtain;

$$
\left\|\sum_{i=1}^{2} M_{T_{i}, S_{i}}\right\| \geq \sup _{\lambda_{i} \in W_{0}\left(S_{i}\right)}\left\{\sup _{\|y\|=1}\left\{\left\|\sum_{i=1}^{2} \lambda_{i} T_{i} y\right\|\right\}\right\}=\sup _{\lambda_{i} \in W_{0}\left(S_{i}\right)}\left\{\left\|\sum_{i=1}^{2} \lambda_{i} T_{i}\right\|\right\} .
$$

Thus, $\quad\left\|\sum_{i=1}^{2} M_{T_{i}, S_{i}}\right\| \geq \sup _{\lambda_{i} \in W_{0}\left(S_{i}\right)}\left\{\left\|\sum_{i=1}^{2} \lambda_{i} T_{i}\right\|\right\} \quad, \quad$ or $\left\|E_{2}\right\| \geq \sup _{\lambda_{i} \in W_{0}\left(s_{i}\right)}\left\{\left\|\sum_{i=1}^{2} \lambda_{i} T_{i}\right\|\right\}$, and this completes the proof.

In the next theorem, the condition necessary for the norm of the elementary operator $E_{2}$ to be equal to the sum of the product of the norms of the corresponding coefficient operators in its definition is given.

Theorem 3.6 Let $E_{2}$ be an elementary operator on $B(H)$ and $S_{i}, T_{i} \in B(H)$ for $i=1,2$. If $\left\|S_{i}\right\| \in W_{0}\left(S_{i}\right)$ and $\left\|T_{i}\right\| \in$ $W_{0}\left(T_{i}\right)$ for $i=1,2$, then;

$$
\left\|E_{2}\right\|=\sum_{i=1}^{2}\left\|T_{i}\right\|\left\|S_{i}\right\| .
$$

Proof. Let $\left\{x_{n}\right\}_{n>1}$ be a sequence of unit vectors on $H$ and $x_{n} \otimes x_{n} \in B(H)$ be a rank one operator on $H$ for a unit vector $x_{n} \in H$, defined by $\left(x_{n} \otimes x_{n}\right)(y)=\left\langle y, x_{n}\right\rangle x_{n}$, for all $\mathrm{y} \in H$.

If $S_{i} \in W_{0}\left(S_{i}\right)$ and $T_{i} \in W_{0}\left(T_{i}\right)$, for $i=1,2$, then there is 
a sequence $\left\{x_{n}\right\}_{n \geq 1}$ of unit vectors in $H$ such that $\lim _{n \rightarrow \infty}\left\langle S_{i} x_{n}, x_{n}\right\rangle=S_{i}, \lim _{n \rightarrow \infty}\left\|S_{i} x_{n}\right\|=\|i\|$ for $i=1,2$, and there is a sequence $\left\{y_{n}\right\}_{n>1}$ of unit vectors in $H$ such that $\lim _{n \rightarrow \infty}\left\langle T_{i} y_{n}, y_{n}\right\rangle=T_{i}, \lim _{n \rightarrow \infty}\left\|T_{i} y_{n}\right\|=\left\|T_{i}\right\|$, for $i=1,2$.

Now, we have;

$$
\begin{aligned}
& \left\|\left(E_{2}\left(x_{n} \otimes x_{n}\right)\right) x_{n}\right\|=\left\|\left(\sum_{i=1}^{2} M_{T_{i}, S_{i}}\left(x_{n} \otimes x_{n}\right)\right) x_{n}\right\| \\
& \leq\left\|\sum_{i=1}^{2} M_{T_{i}, S_{i}}\left(x_{n} \otimes x_{n}\right)\right\|\left\|x_{n}\right\|
\end{aligned}
$$$$
\left\|\sum_{i=1}^{2} M_{T_{i}, S_{i}}\right\|^{2} \geq\left\|\left(\sum_{i=1}^{2} M_{T_{i}, S_{i}}\left(x_{n} \otimes x_{n}\right)\right) x_{n}\right\|^{2}
$$$$
=\left\|\left(T_{1}\left(x_{n} \otimes x_{n}\right) S_{1}+T_{2}\left(x_{n} \otimes x_{n}\right) S_{2}\right) x_{n}\right\|^{2}
$$$$
=\left\|T_{1}\left(x_{n} \otimes x_{n}\right) S_{1} x_{n}+T_{2}\left(x_{n} \otimes x_{n}\right) S_{2} x_{n}\right\|^{2}
$$$$
=\left\|\left\langle S_{1} x_{n}, x_{n}\right\rangle T_{1} x_{n}+\left\langle S_{2} x_{n}, x_{n}\right\rangle T_{2} x_{n}\right\|^{2}
$$$$
=\left\langle\left\langle S_{1} x_{n}, x_{n}\right\rangle T_{1} x_{n}+\left\langle S_{2} x_{n}, x_{n}\right\rangle T_{2} x_{n},\left\langle S_{1} x_{n}, x_{n}\right\rangle T_{1} x_{n}+\left\langle S_{2} x_{n}, x_{n}\right\rangle T_{2} x_{n}\right\rangle
$$$$
=\left\|\left\langle S_{1} x_{n}, x_{n}\right\rangle T_{1} x_{n}\right\|^{2}+\left\|\left\langle S_{2} x_{n}, x_{n}\right\rangle T_{2} x_{n}\right\|^{2}+2 \operatorname{Re}\left\langle\left\langle S_{2} x_{n}, x_{n}\right\rangle T_{2} x_{n},\left\langle S_{1} x_{n}, x_{n}\right\rangle T_{1} x_{n}\right\rangle
$$$$
=\left|\left\langle S_{1} x_{n}, x_{n}\right\rangle\right|^{2}\left\|T_{1} x_{n}\right\|^{2}+\left|\left\langle S_{2} x_{n}, x_{n}\right\rangle\right|^{2}\left\|T_{2} x_{n}\right\|^{2}+2 R e\left\{\left\langle S_{2} x_{n}, x_{n}\right\rangle\left\langle T_{2} x_{n}, T_{1} x_{n}\right\rangle\left\langle S_{1} x_{n}, x_{n}\right\rangle\right\} \text {. }
$$

So, we have;

Now, by the CBS inequality, we have that;

$$
\left|\left\langle T_{2} x_{n}, T_{1} x_{n}\right\rangle\right| \leq\left\|T_{2} x_{n}\right\|\left\|T_{1} x_{n}\right\| \leq\left\|T_{2}\right\|\left\|T_{1}\right\|,
$$

and hence,

$$
\lim _{n \rightarrow \infty}\left\langle T_{2} x_{n}, T_{1} x_{n}\right\rangle=\left\|T_{2}\right\|\left\|T_{1}\right\|
$$

Therefore, taking limits as $n \rightarrow \infty$, we obtain;

$$
\left\|\sum_{i=1}^{2} M_{T_{i}, S_{i}}\right\|^{2} \geq\left\|S_{1}\right\|^{2}\left\|T_{1}\right\|^{2}+\left\|S_{2}\right\|^{2}\left\|T_{2}\right\|^{2}+2\left\|S_{2}\right\|^{2}\left\|T_{2}\right\|^{2}\left\|S_{1}\right\|^{2}\left\|T_{1}\right\|^{2} .
$$
have;

The right hand side is equal to $\left(\sum_{i=1}^{2}\left\|S_{i}\right\|\left\|T_{i}\right\|\right)^{2}$. Thus we

$$
\sum_{i=1}^{2} M_{T_{i}, S_{i}} \geq \sum_{i=1}^{2}\left\|S_{i}\right\|\left\|T_{i}\right\|
$$

Now, since the inequality $\sum_{i=1}^{2} M_{T_{i}, S_{i}} \leq \sum_{i=1}^{2}\left\|S_{i}\right\|\left\|T_{i}\right\|$ always hold (by the triangular inequality), then we obtain $\sum_{i=1}^{2} M_{T_{i}, S_{i}}=\sum_{i=1}^{2}\left\|S_{i}\right\|\left\|T_{i}\right\|$.

\section{Conclusions}

In this paper, we have determined the lower bound of the norm of an elementary operator of length two in a $C^{*}$ algebra $B(H)$ and using the Stampfli's maximal numerical range. The conditions in which this norm is equal to the sum of the products of the corresponding coefficient operators has also been considered. One may attempt this problem for an elementary operator consisting of more that two terms.

\section{References}

[1] D. N. King'ang'i, On the norm of elementary operator of length two, International Journal of Scientific and Innovative Mathematical Research 5 (2017), 34-38.

[2] M. Baraa and M. Boumazgour, A lower bound of the norm of the operator $x \mapsto a x b+b x a$, Extractamathematicae 16 (2001), 223-227.

[3] M. Cabrera and A. Rodriguez, Non-degenerate ultraprimejordan-banach algebras: a zelmanorian treatment, Proc. london. math. soc 69 (1994), 576-604.

[4] D. N. Kingangi, J. O. Agure and F. O. Nyamwala, On the norm of elementary operator, Advances in Pure Mathematics 4 (2014), 309-316.

[5] M. Mathew, More properties of the product of two derivations of a $c^{*}$-algebras, Bull. austral. math. soc 42 (1990), 115-120. 
[6] L. L. Stacho and B. Zalar, On the norm of jordan elementary operators in standard operator algebras, Publ. math. debreen 49 (1996), 127-134.

[7] B. A. Odero, J. A. Ogonji, G. K. Rao, Norms of tensor product elementary operators, International Journal of Multidisciplinary sciences and Engineering 6 (2015), 29-32.

[8] D. R. Jocic et al, Norm inequalities for elementary operators and other inner product type integral transformers with the spectra contained in the unit disc, Filomat 31 (2017), 197206.

[9] A. M. Wafula, N. B. Okelo, O. Ongati, Norms of normally represented elementary operators, International Journal of Modern Science and Technology 3 (2018), 10-26.

[10] S. Paul and C. Gu, Tensor splitting properties of n-inverse pairs of operators, arXiv, 2015, 1-20. 